\title{
EFECTO DE TRES FRECUENCIAS DE ALIMENTACIÓN EN EL CRECIMIENTO, UTILIZACIÓN DE ALIMENTO Y SOBREVIVENCIA DE J UVENILES DE DONCELLA Pseudoplatystoma fasciatum (Linnaeus, 1766).
}

\author{
Franco GUERRA GRANDEZ ${ }^{2}$, Fabiola LOZANO ANCANI ${ }^{1,2}$, Carmen GARCÍA-DÁVILA ${ }^{1,2}$, Luciano \\ RODRÍGUEZ CHU ${ }^{1,2}$, Rosana CUBAS GUERRA ${ }^{1}$, David PANDURO TAFUR ${ }^{1,3}$, Fred CHU-KOO ${ }^{1,2}$
}

1 Universidad Nacional de la Amazonía Peruana - UNAP. Escuela de Post Grado. Cátedra CONCYTEC. Maestría en Acuicultura. Iquitos, Perú.

2 Instituto de Investigaciones de la Amazonía Peruana (IIAP). Programa para el Uso y Conservación del Agua y sus Recursos (AQUAREC). Carretera lquitos - Nauta, Km. 4.5. Iquitos, Perú. E.mail : fchuk20@ gmail.com

3 Dirección Regional de la Producción de Loreto. Programa de Créditos Acuícolas y Pesqueros. Av. Ramirez Hurtado s/n. Iquitos, Perú.

\section{RESUMEN}

La doncella Pseudoplatystoma fasciatum (Linnaeus, 1766), especie que tiene una amplia distribución en Sudamérica, es un pez, que por la calidad de su carne y su rápido crecimiento viene despertando el interés del sector acuícola, principalmente en países como Brasil, Colombia y el Perú. La agencia gubernamental peruana PROMPERU, viene buscando mercados para la carne de este pez y al mismo tiempo articulando con otros actores, el desarrollo de planes e investigaciones para el establecimiento de una oferta exportable de su filete. Uno de estos actores, es el Instituto de Investigaciones de la Amazonía Peruana-IIAP, institución que viene desarrollando una serie de estudios que lleven a la generación de tecnologías para el cultivo de la doncella. El objetivo del presente trabajo fue evaluar los posibles efectos de tres frecuencias de alimentación (FA2, FA4 y FA6) sobre el crecimiento, utilización del alimento y sobrevivencia de juveniles de doncella ( $P$. fasciatum) alimentados con una dieta peletizada (42\% PB) durante 45 días. Al final del estudio, no se registraron diferencias significativas $(\mathrm{P}>0.05)$ en los índices de crecimiento, utilización de alimento, ni en los niveles de sobrevivencia de los peces como efecto de la aplicación de las tres frecuencias de alimentación (2, 4 y 6 veces/día). En conclusión, juveniles de doncella, de $20 \mathrm{~g}$ de peso promedio, sólo necesitarían ser alimentados dos veces al día, lo que permitirá al acuicultor obtener un adecuado rendimiento productivo de los peces, con un mínimo requerimiento de mano de obra.

PALABRAS CLAVE: doncella, Pseudoplatystoma fasciatum, frecuencia de alimentación, crecimiento.

\section{EFFECTS OF THREE FEEDING FREQUENCIES ON GROWTH, FEED UTILIZATION AND SURVIVAL OF TIGER SHOVELN OSE CATFISH Pseudoplatystoma fasciatum J UVENILES}

\begin{abstract}
The tiger shovelnose catfish Pseudoplatystoma fasciatum (Linnaeus, 1766), a species widely distributed in South America, is a fish that due to its high-quality meat and fast growth, has been arising the interest of fish farmers, specially in countries like Brazil, Colombia, and Peru. The Peruvian governmental agency known as PROMPERU, has been looking for new international markets for this fish overseas, and at the same time linking efforts with other Peruvian organisms in order to develop strategies and studies that can lead to the establishment of a sustainable meat production of tiger shovelnose catfish, for international trade. One of these institutions is the Peruvian Amazon Research Institute-IIAP, which is developing a series of studies in order to generate culture technology for this catfish. The main goal of this study was to evaluate the effects of three feeding frequencies (FA2, FA4, and FA6) on the growth, feed utilization and survival of tiger shovelnose catfish $(P$. fasciatum) juveniles fed a pelleted diet $(42 \% \mathrm{CP})$ during 45 days. At the end of the study, no significant differences $(\mathrm{P}>0.05)$ in fish growth, feed utilization and survival levels were recorded as result of the three feeding frequencies (2, 4 and 6 times/day) evaluated. To sum up, tiger shovelnose catfish juveniles of around $20 \mathrm{~g}$ of mean body weight would only need to be fed twice per day, which will allow the fish farmers to obtain an adequate fish yield performance with minimal work requirement.
\end{abstract}

KEYW ORDS: tiger shovelnose catfish, Pseudoplatystoma fasciatum, feeding frequency, growth. 


\section{INTRODUCCIÓN}

Pseudoplatystoma (Bleeker, 1862) es un género que agrupa a tres especies de grandes bagres muy reconocidas en Sudamérica: P. fasciatum, P. tigrinum y $P$. corruscans y de creciente importancia para la acuicultura (Núñez et al., 2008). Estos bagres alcanzan tallas que sobrepasan los $1.3 \mathrm{~m}$, viven en diversos hábitats tales como grandes ríos, lagos y bosques inundados del neotrópico (Reid, 1983) y tienen un reconocido comportamiento migratorio (Barthem \& Goulding, 1997; Loubens \& Panfili, 2000). Su pigmentación es distintiva y consiste de bandas verticales oscuras y pálidas, bandas reticuladas y manchas circulares oscuras. Actualmente se considera que las poblaciones naturales de Pseudoplatystoma están amenazadas debido a la sobreexplotación, construcción de hidroeléctricas, minería, deforestación y contaminación (Carolsfeld et al., 2003).

Desde el año 2007, el IIAP junto al IRD (Francia) vienen ejecutando diversos estudios enfocados en mejorar las técnicas de larvicultura y alevinaje con el propósito de incrementar la sobrevivencia, generar tecnologías para la producción de alevinos en acuicultura y así, fomentar el cultivo de doncella e indirectamente reducir el impacto sobre sus poblaciones naturales. En ese sentido, el adecuado manejo alimenticio de las crías es uno de los principales requisitos a tener en cuenta al momento de desarrollar un paquete tecnológico de cultivo para cualquier especie en acuicultura. La frecuencia de alimentación óptima para los alevinos de doncella aún no ha sido definida y eso conduce a una incertidumbre al momento de diseñar una rutina de alimentación para estos peces.

Es ampliamente conocido que la subalimentación y la sobrealimentación, son factores que pueden ser perjudiciales para la salud de los peces porque causan un marcado deterioro en la calidad del agua, mala utilización del alimento y un incremento en la susceptibilidad a enfermedades (Priestley et al., 2006). Consecuentemente, las tasas de crecimiento específico y de conversión alimenticia, pueden relacionarse directamente a la cantidad de ración y a la frecuencia de alimentación empleada. Por tanto, es importante ser capaces de predecir la frecuencia alimenticia más favorable para la especie y para el tamaño del pez que va a ser cultivado (Priestley et al., 2006). En ese sentido, el presente trabajo evaluó los efectos del uso de tres frecuencias alimenticias en el crecimiento y utilización del alimento en juveniles de doncella.

\section{ATERIALES Y MÉTODOS}

\section{LUGAR DEESTUDIO}

El estudio se realizó en las instalaciones piscícolas del Instituto de Investigaciones de la Amazonía Peruana (IIAP), situado en la comunidad de Quistococha (Km. 4.5 de la carretera Iquitos Nauta), provincia de Maynas, departamento de Loreto.

\section{ORIGEN DE LOS PECES Y PERIODO EXPERIM ENTAL}

El presente trabajo de investigación tuvo un período experimental de 45 días y se empleó un total de 45 alevines de doncella (Pseudoplatystoma fasciatum) obtenidos a través de reproducción inducida de adultos de esta especie (Padilla et al., 2001). Los peces tuvieron pesos homogéneos $(20 \mathrm{~g})$ y fueron previamente adaptados al consumo de alimento balanceado, siguiendo protocolos establecidos en el IIAP.

\section{UNIDADES EXPERIMENTALES}

El experimento se llevó a cabo en nueve (9) tanques rectangulares de cemento $(0.75 \times 0.35 \times 0.45 \mathrm{~m})$ revestidos internamente de mayólicas, los mismos que contaron con aireación independiente y piedras difusoras. El volumen aproximado de agua utilizado en cada unidad experimental fue de 50 litros (densidad de cultivo 1 pez/10 litros), con flujo constante y una tasa de recambio de agua de 1 litro/minuto para así mantener la calidad del agua dentro de los parámetros permisibles para el cultivo de peces y evitar la aparición de patógenos.

\section{DISEÑO EXPERIM ENTAL}

Los 45 peces fueron distribuidos en grupos de cinco (5) individuos entre las nueve (9) unidades experimentales. Tres frecuencias de alimentación (FA2: 2 veces al día, FA4: 4 veces al día y FA6: 6 veces al día), fueron aleatoriamente distribuidos por triplicado en los nueve tanques. Previo al inicio del experimento, los peces fueron sometidos a un periodo de adaptación a sus respectivos tratamientos por espacio de diez días.

Los peces fueron alimentados con una dieta comercial extruída con un tenor proteico de $42 \%$ y $3 \mathrm{~mm}$ de diámetro del pellet, formulada por investigadores del IIAP. La tasa de alimentación empleada fue equivalente al 5\% de la biomasa presente en cada tanque, tasa que se mantuvo hasta el final del estudio. 
La fase experimental tuvo una duración de 45 días y los horarios de alimentación de acuerdo a cada frecuencia fueron los siguientes:

- FA2: 8.00 y $16.00 \mathrm{~h}$.

- FA $4: 8.00,10.30,13.30$ y $17.00 \mathrm{~h}$

- FA6: $8.00,10.00,12.00,14.00,16.00$ y $18.00 \mathrm{~h}$

El alimento que al cabo de una hora no fuera consumido por los peces, fue retirado de los tanques usando el método del sifoneo para posteriormente ser secado en una estufa a $65^{\circ} \mathrm{C}$ por 24 horas, pesado y finalmente restado de los registros de alimentación, a fin de calcular el consumo real de alimento en cada tanque y reajustar los índices de utilización de alimento.

\section{EVALUACIÓN DEL CRECIMIENTO, UTILIZACIÓN DEL} ALIM ENTOY LA SOBREVIVENCIA

Cada quince días se realizaron muestreos biométricos para evaluar el crecimiento en peso y longitud de todos los peces y reajustar las raciones de cada pecera para los próximos 14 días de alimentación. Los índices zootécnicos evaluados fueron los siguientes: tasa de crecimiento específico (TCE), tasa de crecimiento relativo (TCR), tasa de conversión alimenticia (TCA), eficiencia alimenticia (EA), tasa de eficiencia proteica (TEP) y sobrevivencia (S).

\section{CALIDADDEAGUA}

Diariamente se registró la temperatura y el pH del agua utilizando pH metro WTW 330i® y los niveles de oxígeno disuelto con la ayuda de un Oxímetro YSI 55®. Cada quince días se evaluó los niveles de alcalinidad total, amonio, dureza total, dióxido de carbono y nitritos, empleando un KIT AQ-2® de la empresa LaMotte.

\section{ANÁLISIS DE LOS DATOS}

El procesamiento de los datos se realizó en el programa estadístico JMP IN versión 4.0.4 (Sall et al., 2001). Los datos fueron analizados a través de análisis de varianza (One-way ANOVA) teniendo en cuenta de que los valores expresados en porcentajes sean transformados por el método del arco seno previo a su análisis en ANOVA. Los resultados son mostrados como el promedio \pm la desviación estándar(DS).

\section{RESULTADOS}

\section{CALIDADDELAGUA}

En líneas generales, los principales parámetros físicos y químicos del agua se mantuvieron dentro de lo esperado, es decir dentro de los límites adecuados para el cultivo de peces amazónicos como la doncella (Tabla 1).

\section{CRECIMIENTO, UTILIZACIÓN DEL ALIMENTO $Y$ SOBREVIVENCIA}

En la Tabla 2, se muestran los principales índices de crecimiento, utilización del alimento y los niveles de sobrevivencia obtenidos de los ejemplares de doncella criados durante los 45 días de experimentación bajo la influencia de tres frecuencias de alimentación. Los indicadores de crecimiento en peso (PF, GP, GPD, TCE, TCR, BF y BG) no mostraron diferencias significativas entre los tratamientos evaluados $(\mathrm{P}>0.05)$. El análisis de varianza tampoco registró diferencias $(\mathrm{P}>0.05)$ significativas en lo que respecta al consumo (alimento consumido y proteína consumida) y a la utilización del alimento (TCA, EA y TEP) entre los peces sometidos a las tres frecuencias de alimentación empleadas en el presente estudio (Tabla 2). A pesar de que se registró la muerte de dos ejemplares en el tratamiento FA6, no hubo diferencias significativas $(\mathrm{P}>0.05)$ en cuando a los niveles de sobrevivencia $(\mathrm{FA} 2=100 \%, \mathrm{FA} 4=100 \%$ y FA6 $=$ $86.67 \pm 11.55 \%)$. 
Tabla 1. Calidad de agua (promedio \pm desviación estándar) registrada durante la fase de cultivo de 45 juveniles de doncella Pseudoplatystoma fasciatum alimentados con tres frecuencias alimenticias (FA2: 2 veces/día, FA4: 4 veces/día y FA6: 6 veces/día) durante 45 días.

\begin{tabular}{l|c|c|c}
\hline \multirow{2}{*}{ PARÁm ETROS } & \multicolumn{3}{c}{ (Promedio \pm desviación estándar) } \\
\cline { 2 - 4 } & FA2 & FA4 & FA6 \\
\hline Temperatura $(\mathrm{o}$ C) & $26.2 \pm 0.96$ & $26.4 \pm 1.12$ & $26.3 \pm 1.41$ \\
Oxígeno disuelto $(\mathrm{mg} / \mathrm{l})$ & $5.73 \pm 0.32$ & $5.61 \pm 0.54$ & $5.7 \pm 0.61$ \\
pH (upH) & $6.54 \pm 0.48$ & $6.6 \pm 0.32$ & $6.65 \pm 0.43$ \\
Nitritos (ppm) & $<0.05$ & $<0.05$ & $<0.05$ \\
Amonio (ppm) & $<0.2$ & $<0.2$ & $<0.2$ \\
Dióxido de carbono (ppm) & $5.5 \pm 3.5$ & $4.5 \pm 3.0$ & $3.5 \pm 3.0$ \\
Dureza total (ppm) & $12.0 \pm 3.0$ & $14.0 \pm 2.0$ & $14.0 \pm 3.0$ \\
\hline
\end{tabular}

Tabla 2. Crecimiento e índices zootécnicos (promedio \pm desviación estándar) registrados en juveniles de doncella, Pseudoplatystoma fasciatum, alimentados con tres frecuencias de alimentación (FA2: 2 veces/día, FA4: 4 veces/día y FA6: 6 veces/día) durante 45 días.

\begin{tabular}{l|c|c|c|c}
\hline \multirow{2}{*}{ INDICES ZOOTECNICOS } & \multicolumn{2}{|c|}{ FRECUENCIAS ALIM ENTICIAS } & \multirow{2}{*}{ PROB. } \\
\cline { 2 - 4 } & FA2 & FA4 & FA6 & \\
\hline PI (g) & $20.6 \pm 1.0$ & $20.0 \pm 1.0$ & $19.4 \pm 1.0$ & 0.7140 \\
\cline { 1 - 4 } PF (g) & $32.7 \pm 1.6$ & $32.1 \pm 1.6$ & $30.7 \pm 1.6$ & 0.3639 \\
\hline GP (g) & $12.1 \pm 0.5$ & $12.08 \pm 0.5$ & $11.3 \pm 0.6$ & 0.1883 \\
\hline GPD (g/día) & $0.27 \pm 0.01$ & $0.27 \pm 0.01$ & $0.25 \pm 0.01$ & 0.1883 \\
\hline TCR (\%) & $58.7 \pm 2.7$ & $60.9 \pm 8.7$ & $58.8 \pm 7.2$ & 0.9040 \\
\hline TCE (\%peso/día) & $1.03 \pm 0.04$ & $1.05 \pm 0.12$ & $1.02 \pm 0.1$ & 0.9120 \\
\hline BI (g) & $102.9 \pm 6.2$ & $100.2 \pm 10.31$ & $96.9 \pm 9.2$ & 0.7140 \\
\hline BF (g) & $163.2 \pm 8.1$ & $160.6 \pm 8.1$ & $153.4 \pm 8.0$ & 0.3639 \\
\hline BG (g) & $60.3 \pm 2.4$ & $60.4 \pm 2.4$ & $56.5 \pm 2.9$ & 0.1883 \\
\hline S (\%) & 100 & 100 & $86.7 \pm 11.6$ & 0.0787 \\
\hline EA & $0.42 \pm 0.04$ & $0.47 \pm 0.27$ & $0.42 \pm 0.11$ & 0.9079 \\
\hline TCA & $2.37 \pm 0.4$ & $2.46 \pm 1.0$ & $2.47 \pm 0.6$ & 0.9779 \\
\hline TEP & $0.59 \pm 0.07$ & $0.66 \pm 0.35$ & $0.71 \pm 0.42$ & 0.9077 \\
\hline Proteína consumida (g) & $59.8 \pm 2.8$ & $61.8 \pm 23.5$ & $58.3 \pm 11.3$ & 0.9618 \\
\hline Alimento consumido (g) & $142.5 \pm 6.6$ & $147.0 \pm 55.8$ & $138.8 \pm 26.9$ & 0.9618 \\
\hline
\end{tabular}

\section{Leyenda:}

$\mathrm{PI}=$ peso inicial, $\mathrm{PF}=$ peso final, $\mathrm{GP}=$ ganancia de peso, $\mathrm{GPD}=$ ganancia de peso diario, $\mathrm{BI}=$ biomasa inicial, $\mathrm{BF}=$ biomasa final, $\mathrm{BG}$ = biomasa ganada, $T C R=$ tasa de crecimiento relativo, $T C E=$ tasa de crecimiento específico, EA = eficiencia alimenticia, TCA = tasa de conversión alimenticia, $\mathrm{S}=$ sobrevivencia, $\mathrm{TEP}=$ tasa de eficiencia proteica. 


\section{DISCUSIÓN}

El costo de las raciones alimenticias y las horashombre empleadas en la alimentación son dos de las principales variables que afectan el costo final de las operaciones en acuicultura (Riche et al., 2004). En ese sentido, una de las estrategias comúnmente utilizadas para maximizar el crecimiento de los organismos en cultivo es justamente, maximizar la alimentación. Sin embargo, ha quedado demostrado que esta estrategia tiende a incrementar el alimento que se pierde en el estanque (entre 5-30\% de lo ofertado), hasta significar casi el 50\% de los desperdicios sólidos producidos en el proceso (Cho et al., 1991). Consecuentemente, este tipo de manejo resulta en la disminución de la eficiencia alimenticia, deterioro de la calidad del agua y en el aumento de los costos de operación. Por tanto, un buen manejo de la alimentación en todas las fases de cultivo, que incluya una apropiada frecuencia de alimentación, reducirá la sobrealimentación y maximizará la eficiencia alimenticia.

De acuerdo a nuestros resultados, los parámetros de crecimiento, utilización de alimento, así como la sobrevivencia de los juveniles de doncella fueron similares en los tres tratamientos evaluados. Similares resultados fueron reportados por Gandra et al. (2007) en juveniles de paiche (Arapaima gigas). Sin embargo, hay experiencias reportadas en la literatura donde el uso de distintas frecuencias de alimentación si influyen decisivamente en el desempeño productivo de los organismos en cultivo. Por ejemplo, en un estudio similar, Ferreira et al. (2007), aplicaron las mismas frecuencias de alimentación evaluadas en el presente estudio (2, 4 y 6 veces al día) en la alimentación de alevinos de "dourado" (Salminus brasiliensis) y observaron que, al cabo de 35 días, hubo una marcada diferencia en la cantidad de alimento consumido, la velocidad de crecimiento (TCE) y los niveles de sobrevivencia en los peces alimentados con la frecuencia de alimentación de seis veces al día. Similar resultado también fue reportado por Wang et al. (1998), en el consumo de alimento en ejemplares híbridos de "sunfish" (Lepomis cyanellus $\mathrm{x}$ L. macrochirus) alimentados seis veces al día.

Definitivamente, el contar con información relevante sobre los regimenes u horarios de alimentación de una especie en cultivo es importante para alcanzar una eficiente producción y para asegurar las mejores tasas de conversión alimenticia y ganancia de peso. Por eso, un paso importante en la estrategia alimenticia es determinar la frecuencia de alimentación óptima. Otro claro ejemplo de la influencia del empleo de distintas frecuencias de alimentación en el desempeño productivo de un organismo; es el trabajo de Ruohonen et al. (1998), quienes al emplear tres frecuencias de alimentación (1, 2 y 4 veces/día) en la trucha arco iris (Oncorhynchus mykiss) encontraron que, para maximizar el desempeño productivo de esta especie, sería ideal alimentarla 4 veces/día.

Revisando la literatura relacionada a la temática del estudio, pudimos darnos cuenta de la importancia de este trabajo. Existe una severa escasez de información de estudios similares realizados en doncella; por lo que nos vimos obligados a discutir nuestros resultados con estudios similares realizados en otros bagres del mundo. Así, encontramos que Webster et al. (2001), concluyeron que en el bagre de canal (Ictalurus punctatus), una especie oriunda de Norteamérica y ampliamente cultivada en el sur de Estados Unidos, es posible mejorar la eficiencia alimenticia, la tasa de crecimiento y disminuir los residuos de alimento empleando una adecuada frecuencia de alimentación. De igual modo, Canton et al. (2007), evaluaron el uso de cuatro frecuencias alimenticias (1,2,3 y 4 veces al día) en la alimentación de juveniles de jundiá (Rhamdia quelen), un bagre de importancia comercial del sur de Brasil, cultivado en estanques de tierra y alimentado con una dieta extrusada ( $42 \%$ proteína); observando que los peces alimentados cuatro veces al día presentaron valores de TCE superiores, siendo esa tasa significativamente diferente cuando fue comparada con los peces alimentados una vez al día.

Estudios realizados en carácidos, como el de Hayashi et al. (2004), indican que la ganancia de peso de alevinos de lambari (Astyanax bimaculatus), alimentados cuatro veces al día, fue superior a la de los peces alimentados dos, seis y ocho veces al día. Feiden et al. (2007), reportan que la frecuencia alimenticia más eficiente a ser empleada en los alevinos de lisa (Leporinus elongatus) es la de cuatro veces al día, puesto que produce una mejor ganancia de peso.

Aparentemente, los peces de los tratamientos FA2 y FA4 consumieron más alimento que los del tratamiento FA6; sin embargo, estas diferencias no fueron significativas, a diferencia de otros estudios donde a medida que se incrementa las frecuencias de alimentación también aumenta el consumo de alimento (Wang et al., 1998; Başçinar et al., 2007).

En los 45 días de cultivo, la tasa de conversión alimenticia varió entre 2.37 y 2.47 , y la eficiencia alimenticia entre 42 y $47 \%$, valores que indican una baja asimilación del alimento. Sin embargo, siendo la doncella un pez eminentemente carnívoro y al que todavía se le viene adaptando al consumo de dietas secas, entonces es absolutamente normal que el grado de asimilación del alimento inerte sea particularmente menor que en otras especies amazónicas de hábitos omnívoros, que presentan mayor adaptabilidad al alimento balanceado, como son las casos específicos de la gamitana (Colossoma macropomum) y el paco (Piaractus brachypomus). 
La sobrevivencia promedio observada en los juveniles de doncella durante el experimento varió entre 86.7 y $100 \%$, que son niveles similares a los reportados por Ali et al. (2005) y Khan et al. (2009) y algo mayores a los reportados por Azad et al. (2004) en el bagre asiático Pangasius hypophthalmus, una especie de alto valor comercial y que actualmente predomina en el sudeste asiático, principalmente en Vietnam. Los altos niveles de sobrevivencia observados en $P$. fasciatum podría deberse al adecuado tamaño al momento de la siembra $(20 \mathrm{~g})$.

En conclusión, los resultados del presente trabajo muestran que la frecuencia de alimentación no tuvo influencia en el crecimiento ni en la utilización del alimento. Esto significa que da lo mismo emplear cualquiera de las frecuencias evaluadas en el estudio, puesto que estadísticamente producirán resultados similares. Sin embargo, para fines prácticos en cuanto al manejo de la alimentación de juveniles de $P$. fasciatum, se sugiere el uso de la frecuencia FA2 (dos veces al día) o en todo caso, que la alimentación manual no supere el límite máximo de cuatro veces al día, puesto que permitirá obtener similar rendimiento productivo con mínimo requerimiento de mano de obra.

\section{AGRADECIMIENTOS}

Al Proyecto INCAGRO, que a través del Contrato $\mathrm{N}^{\circ}$ 2007-0542-AG-INCAGRO/FDSE, subproyecto "Mejoramiento genético y producción intensiva de alevinos seleccionados de doncella Pseudoplatystoma fasciatum (Linnaeus, 1776) en la Amazonía peruana" financió la ejecución del presente estudio.

\section{BIB LIOGRAFÍA CITADA}

Ali, M.Z.; Hossain, M.A.; Gazid, M.A. 2005. Effect of mixed feeding schedules with varying dietary protein levels on the growth of cutch catfish, Pangasius hypophthalmus (Sauvage) with silver carp, Hypophthalmichthys molitrix (Valenciennes) in ponds. Aquaculture Research, 36:627-634.

Azad, M.A.K.; Rahman, M. R.; Rahman, Z.; Kader, M.A.; Haque, M.M.; Alam, M.J. 2004. Polyculture of carp, tilapia and pangas using low cost inputs. Pak. J. Biol. Sci., 7(11):1918-1926.

Başçinar, N.; Çakmak, E.; Çavdar, Y.; Aksungur, N. 2007. The effect of feeding frequency on growth performance and feed conversion rate of Black sea trout (Salmo trutta labrax Pallas, 1811). Tur. J. Fisheries. Aqua. Sci., 7: 13-17.

Barthem, R. B.; Goulding, M. 1997. Os bagres balizadores: ecologia, migração de pexies a mazônicos. Soci e d a d C ivil Mamirahuá/MTC-CNPq/IPAAM.
Canton, R.; Weingartner, M.; Fracalossi, D.M.; Zaniboni-Filho, E. 2007. Influência da freqüência alimentar no desempenho de juvenis de jundiá. Revista Brasileira de Zootecnia, 36(4):749-753.

Carolsfeld, J.; Harvey, B.; Ross, C.; Baer, A. 2003. Migratory Fishes of South America. Biology fisheries and conservation status. $372 \mathrm{pp}$.

Cho, C.Y.; Hynes, J.D.; Wood, K.R.; Yoshida, H.K. 1991. Quantitation of fish culture wastes by biological (nutritional) methods: the development of high nutrient dense (HND) diets. In: Cowey, H.D.; Cho, C.Y. (Eds). Nutritional Strategies and Aquaculture Waste. Proceedings of the First International Symposium on Nutritional Strategies in Management of Aquaculture Waste. Univ. Guelph, Ontario, Canada. pp.37-49.

Feiden, A.; Zaminhan, M.; Azambuja de Freitas, J.M.; Lui, T.A.; dos Reis E.S.; Boscolo, W.R. 2007. Freqüência de arraçoamento para alevinos de Piaparas Leporinus elongatus. In: $1^{\circ}$ Congresso Brasileiro de Produção de Peixes Nativos de Água Doce. EMBRAPA. Mato Grosso do Sul, 5p.

Ferreira, R. D. A.; Thiesen, R.; Costa, T. R.; Ishikawa, M. M.; Hisano, H. 2007. Frequiência alimentar no desempenho de alevinos de dourado. In: $1^{o}$ Congresso Brasileiro de Produção de Peixes Nativos de Água Doce. EMBRAPA. Mato Grosso do Sul, 5p.

Gandra, A.L.; Ituassu, D.R.; Pereira-Filho, M.; Roubach, R.; Crescencio, R.; Cavero, B.A.S. 2007. Pirarucu growth under different feeding regimes. Aquaculture International, 15:91-96.

Hayashi, C.; Meurer, F.; Boscolo, W.R.; Figueiredo, C.H.L.; Kavata, L.C.B. 2004. Freqüência de arraçoamento para alevinos de lambari do raboamarelo (Astyanax bimaculatus). Revista Brasileira de Zootecnia, 33(1):21-26.

Khan, S.; Hossain, M.S.; Haque, M.M. 2009. Effects of feeding schedule on growth, production and economics of pangasiid catfish (Pangasius hypophthalmus) and silver carp (Hypophthalmichthys molitrix) polyculture. J. Bangladesh Agril. Univ., 7(1):175-181.

Loubens, G.; Panfili, J. 2000. Biologie de Pseudoplatystoma fasciatum et $P$. tigrinum (Teleostei: Pimelodidae) dans le bassin du Mamoré (Amazonie Bolivienne). Ichthyological Exploration of Freshwaters, 11:13-34.

Padilla, P. P.; Alcántara, B. F.; Ismiño, O. R. 2001. Reproducción inducida de la doncella Pseudoplatystoma fasciatum y desarrollo embrionario-larval. Folia Amazónica, 12(1-2): 141-155. 
Priestley, S.M.; Stevenson, A.E.; Alexander, L.G. 2006. The influence of feeding frequency on growth and body condition of the common Goldfish (Carassius auratus). Journal of Nutrition, 136:1979-1981.

Reid, S. 1983. La biología de los bagres rayados Pseudoplatystoma fasciatum Pseudoplatystoma tigrinum en la cuenca del río Apuré, Venezuela. Revista UNELLEZ de Ciencia y Tecnología de Producción Agrícola, 1: 13-41.

Riche, M.; Oetker, M.; Haley, D.I.; Smith, T.; Gfarling, D.L. 2004. Effect of feeding frequency in consumption, growth, and efficiency in juveniles tilapia (Oreochromis niloticus). The Israeli Journal of Aquaculture - Bamidgeh, 56(4):247255.

Ruohonen, K.; Vielma, J.; Grove, D.J. 1998. Effects of feeding frequency on growth and food utilisation of rainbow trout (Oncorhynchus mykiss) fed low fat herring or dry pellets. Aquaculture, 165:111121.
Salinas, C. Y.; Agudelo, C. E.; Alonso, G.J.C.; Prieto, P. E. F.; Bonilla O. 2007. Peces de importancia económica en la cuenca amazónica colombiana. Instituto Amazónico de Investigaciones Científicas. Leticia, Colombia. 70p.

Sall, J.; Lehman, A.; Creighton, L. 2001. JMP Start Statistics: A guide to statistics and data analysis using JMP and JMP IN software. Duxbury Thomson Learning. Canada. 491p.

Wang, N.; Hayward, R.S.; Noltie, D.B. 1998. Effect of feeding frequency on food consumption, growth, size variation, and feeding pattern of age-0 hybrid sunfish. Aquaculture, 165:261-267.

Webster, C.D.; Thompson, K.R.; Morgan, A.M.; Grisby, E.J.; Dasgupta, S. 2001. Frequency feeding affects growth, not fillet composition, of juvenile Sunshine bass Morone chrysops x M. saxatilis grown in cages. Journal of the World Aquaculture Society, 32(1):79-88. 


